\title{
Cervical nystagmus due to loss of cerebellar inhibition on the cervico-ocular reflex: a case report
}

\author{
AM BRONSTEIN, JD HOOD \\ From the Medical Research Council Neuro-Otology Unit, Institute of Neurology, National Hospital for \\ Nervous Diseases, London, UK
}

SUMMARY Studies of the cervico-ocular reflex and the vestibulo-ocular reflex have been carried out separately and in combination on a patient with gait ataxia due to a cerebellar tumour. With the head fixed in space, body rotation to the right (left neck torsion) induced marked nystagmus to the left in darkness. Vestibulo-ocular responses to sinusoidal rotation were symmetrical while the neck was immobilised and asymmetric when it moved freely. It is suggested that the cervical nystagmus seen in this case was the result of removal of cerebellar inhibition upon the cervicoocular reflex and that abnormal interaction of cervical and vestibular inputs could have played a role in the patient's unsteadiness.

While clinical and experimental studies have provided abundant evidence of the inhibitory role played by the cerebellum in the vestibulo-ocular reflex, ${ }^{1-3}$ much less is known about its influence upon the cervico-ocular reflex. This stems in part from the difficulty in eliciting the reflex in normal animals and humans. ${ }^{4}$ In addition, although in certain clinical conditions nystagmus can be elicited on neck torsion, ${ }^{5}$ it is usually difficult to exclude a vascular aetiology. The present paper is concerned with a unique case in which a nystagmus of undoubted cervical origin has been demonstrated due, in all probability, to a derangement of the central connections.

\section{Case report}

Two weeks before admission a 58-year-old male pipe smoker developed occipital headache and difficulty in walking. Initially the latter was only evident on turning when he would sway from side to side. Walking in a straight line was unimpaired. This sympton gradually increased, however, and at the time of his admission he staggered markedly. A few days before admission he developed very mild hand clumsiness and slurred speech. The headaches had also been increasing and he was

AM Bronstein was supported by the Brain Research Trust.

Address for reprint requests: Dr JD Hood, Institute of Neurology, National Hospital for Nervous Diseases, Queen Square, London WC1N 3BG, UK.

Received 11 May 1984 and in revised form 10 July 1984. Accepted 14 July 1984 anorexic and vomited on one occasion.

General examination was unremarkable; blood pressure was $140 / 80 \mathrm{~mm} \mathrm{Hg}$. Neurological examination disclosed aे broad based stance and marked gait ataxia; he was espe $\frac{\stackrel{P}{\Phi}}{\square}$ cially unsteady on turning while walking. Cranial nerves fundi, sensation and muscle power were normal apart frome $T$ diminished pin-prick sensation on the feet. There wa\$్ moderate lower limb ataxia but upper limbs were considered normal. In the lower limbs, deep reflexes were brisk and tone was thought to be marginally increased but plano tar and cutaneous abdominal reflexes were normal. There $e^{+}$ was a moderate dysarthria. On oculomotor examination no palsies were found. No spontaneous nystagmus on primary position of gaze but gaze evoked nystagmus to right, left and upwards was elicited. Lying down, without neck torsion, either with right or left ear down, produced a nonfatiguing nystagmus beating towards the upper ear, without latent period or vertiginous sensation. Extreme neck torsion to right and left in the sitting position did not induced any nystagmus or vertigo. Smooth pursuit was broken up (saccadic) in all directions. Saccades were slightly hypometric, but seemed to have normal speed.

A CT scan showed a cerebellar mass involving the anterior vermis, paramedian region and partial hemisphere on the right (fig 1). An occipital craniectomy was carried out; histological diagnosis of undifferentiated adenocarcinoma was made. A chest radiograph revealed a lung tumour thought to be the origin of the cerebellar metastasis.

Pre-operative investigations
Cervico-ocular reflex (COR) In order to examine the role $\frac{D}{D}$ of the neck reflexes in isolation it is necessary to rotate the trunk while maintaining the head immobile. To this end $N$ the subject sat in a rotating chair which could be moved manually. In this position he was required to bite on a $N$ 


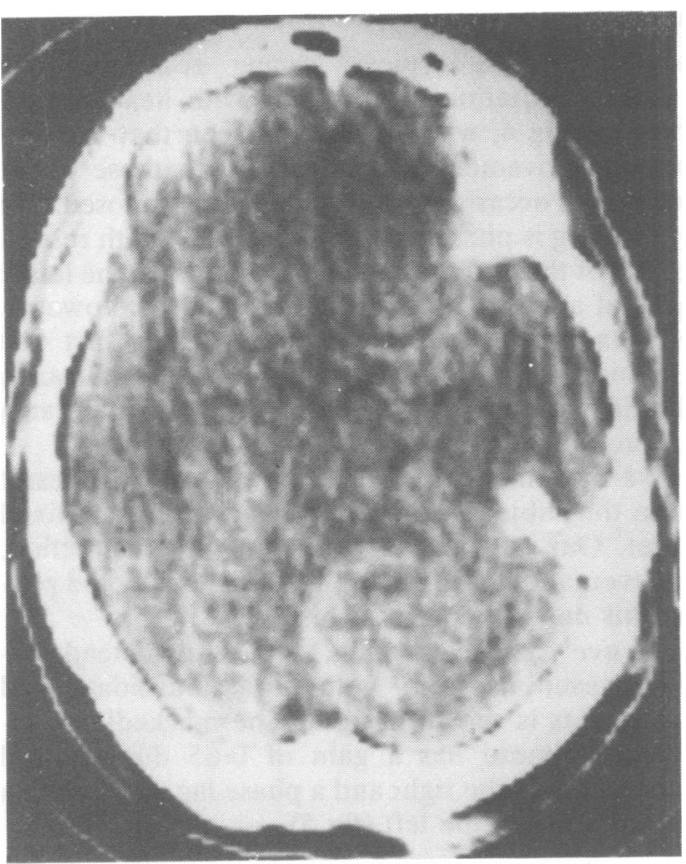

Fig 1 Enhanced CT scan at the level of the superior aspect of the posterior fossa showing rounded abnormal enhancement in the mid line and right cerebellar hemisphere associated with a small amount of oedema. The fourth ventricle was distorted and displaed to the left but the rest of the ventricular system was only mildly dilated.

dental plate attached to a rigid frame mounted on the wall. The chair was housed in a light-proofed room so that where necessary tests could be carried out in total darkness. A target light mounted immediately to the front of the subject in line with primary gaze was presented from time to time thus enabling eye deviation to be monitored throughout the course of the test. A closed circuit TV display using an infra-red camera directed at the subject's head attested to the absence of any inadvertant head movement. Eye movements were recorded with bitemporal DC EOG and calibrated with targets $10^{\circ}$ apart. Chair and eye position, together with a target signal were displayed on an ink-jet recorder.

Testing was carried out in total darkness using both ramp and sinusoidal stimuli. In the case of the former, the chair was moved randomly from left to right or vice versa at a peak velocity of about $12 \% \mathrm{~s}^{-1}$ over varying angular displacements from 16 to $44^{\circ}$. About 3 to 6 seconds following the termination of each discrete movement the target light was presented so that measurements could be made of any residual deviation of the eyes from the primary position of gaze. An interval of 8-10 s separated each movement of the chair. Twenty-one stimuli in all were delivered; four of these were rejected because of head movement monitored on the video screen. Sinusoidal trunk movement was applied manually over a range of frequen- cies from 0.1 to $0.4 \mathrm{~Hz}$ synchronised to a metronome. Vestibulo-ocular reflex (VOR) The vestibulo-ocular reflex was studied under conditions of both passive and active head movement. In the former, the patient with head firmly secured to the chair was rotated sinusoidally in total darkness at a frequency of $0.3 \mathrm{~Hz}$ over a total amplitude of $\pm 25^{\circ}$. Both chair displacement and eye movements were recorded simultaneously. In the case of active head movements the subject, again in total darkness, was instructed to move his head sinusoidally at $0.3 \mathrm{~Hz}$ in time with a metronome. The resultant ocular response thus combined both vestibular and cervical inputs. Head movement was monitored by means of a light helmet attached to a low torque potentiometer.

\section{Results}

\section{Cervico-ocular reflex}

(a) Ramp stimuli Rotation of the trunk, say to the right, resulted effectively in relative movement of the head to the left and for convenience will be referred to as HL; likewise with trunk to left as HR.

All trunk movements to right (HL) irrespective of the starting position, evoked a marked saw-tooth type nystagmus to the left with an exponential decay of the slow phase component reminiscent of gaze evoked nystagmus of cerebellar origin. On cessation of movement, presentation of the target light resulted in an eye movement of about $15^{\circ}$ to the right indicative of a sustained tonic deviation of the eyes to the left. A typical example is shown in fig 2 .

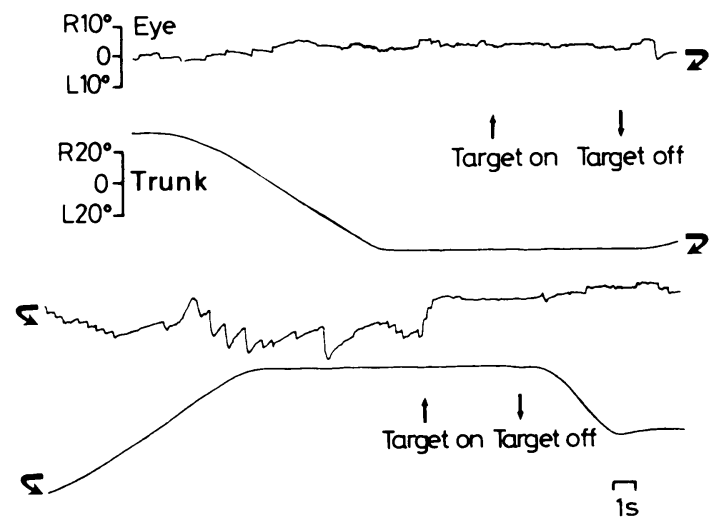

Fig 2 Cervico-ocular reflex-ramp stimuli. Eye movement recordings in darkness with head fixed and trunk rotated. Upper tracing shows minimal nystagmic response to right on trunk rotation from right to left (neck torsion to right). Lower tracing shows marked left beating nystagmus induced by trunk rotation from left to right (neck torsion to left). Vertical arrows indicate presentation of target light in primary gaze position. In the lower tracing this reveals a marked tonic deviation of the eyes to the left in the absence of optic fixation. 


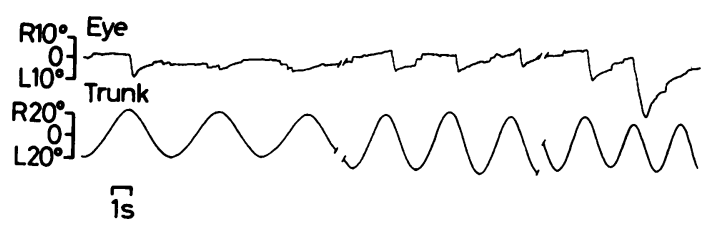

Fig 3 Cervico-ocular reflex-sinusoidal stimuli. Lower tracing shows sinusoidal rotation of trunk with head fixed. Upper tracing shows nystagmic response in darkness. Note that nystagmus to left is triggered predominantly at peak body displacement to right $(H L)$ and increases in amplitude with increasing frequency. Small amplitude nystagmus to right occasionally appears and is likewise phase related to peak trunk displacement $(H R)$.

In contrast, the responses to trunk movement to left (HR) were weak and variable although in six out of ten trials a low amplitude nystagmus to the right appeared which was not dissimilar to responses found in normal subjects. Defining gain as the ratio of peak slow phase velocity to peak chair velocity, a clear asymmetry of the COR is apparent with (HL) gain of $1.40 \pm 0.65$ and a (HR) gain of $0 \cdot 10 \pm 0 \cdot 10$.

(b) Sinusoidal stimuli As shown in fig 3, eye movement responses to sinusoidal movement of the trunk were remarkable in that peak displacement to the right (HL) consistently appeared to trigger large amplitude rapid components to the left followed, in turn, by a slow phase to the right which was most obvious at higher frequencies. Peak displacement to the left (HR) elicited small amplitude saccadic movements to the right.

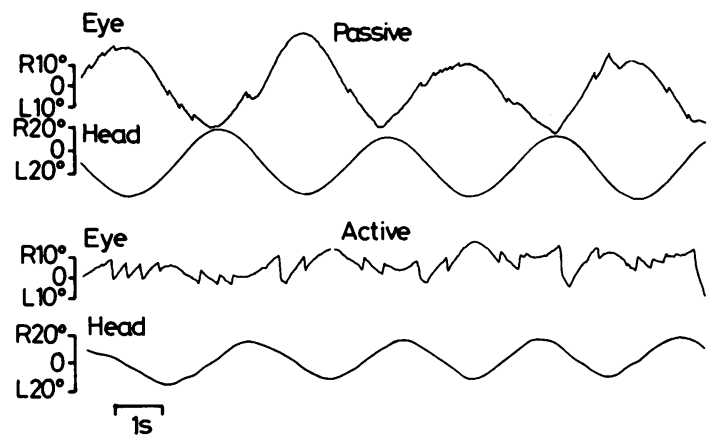

Fig 4 Passive-sinsuoidal rotation of whole body with head secured to chair eliciting vestibular ocular response without cervico-ocular reflex. Eye movements are compensatory to head movements and marginally asymmetric.

Active-voluntary movements of head to left and right eliciting vestibulo-ocular plus cervico-ocular reflex. Lower tracing shows head position monitored by helmet attachment. Note clear directional preponderance to left of nystagmic response in terms of amplitude and slow component velocities.
Vestibulo-Ocular Reflex:

(a) Passive A typical tracing resulting from sinusoidal rotation of the body with head fixed is shown in fig 4 , where it will be seen that the eyes execute movements compensatory to those of the body, with occasional nystagmus superimposed. The eye tracing is phase advanced some $10^{\circ}$ with respect to that of the body with a gain as shown to the left of fig 5 , of about $0 \cdot 80$. It needs to be noted, however, that Barr et $a^{6}$ have commented on the fact that responses to stimuli of this kind can be significantly altered by the particular strategy voluntarily adopted by the subject.

Eye movements of this kind, for example, result when the subject assumes an imaginary earth fixed target. Our patient was given no such instruction, however, and it is highly unlikely that he would pursue this unusual course of his own volition.

(b) Active The eye tracing, together with head position, resulting from voluntary sinusoidal head movements is shown in fig 4 . The markedly asymmetric response has a gain of 0.65 during head movements to the right and a phase lag of $10^{\circ}$ with a gain of unity to the left (fig 5).

\section{Discussion}

The main finding of importance which has emerged from these studies is the appearance of a well marked nystagmus to the left on trunk movement to the right (HL) with head restrained (fig 2). The nystagmus appears initially during the trunk movement and persists for some time after cessation of movement. Adopting similar test procedures a cervical nystagmus of this kind can be readily demonstrated in patients with absent labyrinthine function. ${ }^{78}$ Indeed it is maintained that the resultant gain in the COR plays a compensatory role for the loss of the Vestibulo-ocular reflex (VOR). ${ }^{9}$ On the available evidence, however, it would seem unlikely that the peripheral vestibular apparatus of our patient was in any way impaired. There remains the possibility of a vascular aetiology. This can certainly be called in to account for the so-called cervical nystagmus/vertigo resulting, for example from whiplash injury or vertebro-basilar insufficiency, in which mechanical compression of the vertebral arteries during neck movements can affect blood supply of the labyrinth and the brain stem. ${ }^{10} 11$ If this were the case, however, it is to be expected that it would be most marked on extreme neck torsion; instead all ramp movements were equally effective in eliciting nystagmus whether the movement was from mid-line to HL or HR to mid-line. In the circumstances our conclusion is that the nystagmus is the direct out- 


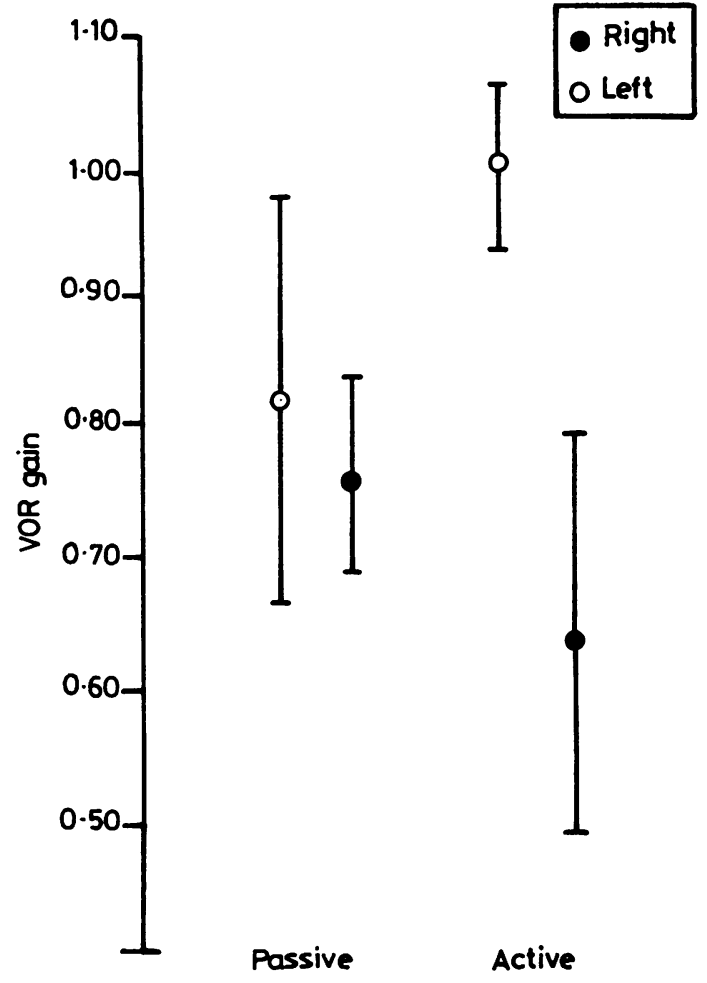

Fig 5 Vestibulo-ocular reflex (slow phase peak eye velocity/head peak velocity) in passive and active conditions at $0.3 \mathrm{~Hz}$ in darkness. Values obtained during head rotation to left and right differ markedly in the active mode. Mean and standard deviations are shown.

come of the cerebellar lesion which in turn implies that the COR is under the inhibitory control of the cerebellum. This is perhaps not surprising since it is well established that the vestibulo-cerebellum exerts a similar inhibitory effect upon the VOR. ${ }^{3}$ According to the CT scan the vestibulo-cerebellum would almost certainly have been spared with the lesion mostly involving anterior and rostral parts of the posterior lobes. These areas, however, are known to receive strong proprioceptive projections including those from the cervical receptors. ${ }^{12-14}$

Apart from the typical gait ataxia which results from lesions in this region our findings suggest that a new factor which needs to be taken into account is abnormal interaction of the cervical and vestibular inputs. The effect of this is best illustrated in the abnormalities found during active head movements exemplified in fig 5 . These in turn could give rise to subtle postural disorders such as the imbalance experienced by our patient on turning while walking.

Although to the best of our knowledge these findings are unique, it is perhaps worth stressing that they have come about largely by the chance application of somewhat sophisticated test procedures to a particular patient during the course of a more general enquiry into the role of cervical proprioception. Ill-defined postural abnormalities related to head movement are not uncommon in patients with central disorders and a more systematic investigation of this kind might well reveal that associated derangements of the kind described here are the rule rather than the exception.

\section{References}

${ }^{1}$ Fernandez C, Frederickson JM. Experimental cerebellar lesions and their effect on vestibular function. Acta Otolaryng (Suppl) 1964;192:52-62.

${ }^{2}$ Zee DS, Yee RD, Cogan DG, Robinson DA, King Engel W. Ocular motor abnormalities in hereditary cerebellar ataxia. Brain 1976;99:207-34.

${ }^{3}$ Wilson VJ, Melvill Jones G. Mammalian Vestibular Physiology. New York: Plenum Press, 1979.

4 Fuller JH. The dynamic neck-eye reflex in mammals. Exp Brain Res 1980;41:29-35.

5 Biemond A, De Jong JMBV. On cervical nystagmus and related disorders. Brain 1969;92:437-58.

- Barr CC, Schulthesis LW, Robinson DA. Voluntary, non-visual control of the human vestibulo-ocular reflex. Acta Otolaryngol 1976;81:365-75.

7 Jongkees LBW. Cervical Vertigo. Laryngoscope 1969; 79: 1473-84.

${ }^{8}$ Kasai T, Zee DS. Eye-head co-ordination in labyrinthine-defective human beings. Brain Res $1978 ; 144: 123-41$.

' Dichgans J, Bizzi E, Morasso P, Tagliasco V. Mechanisms underlying recovery of eye-head co-ordination following bilateral labyrinthectomy in monkeys. Exp Brain Res 1973;18:548-62.

10 Tissington Tatlow WF, Bammer HG. Syndrome of vertebral artery compression. Neurology (Minneap) 1957; 7:331-40.

"Toglia JU. Acute flexion-extension injury of the neck. Neurology (Minneap) 1976;26:808-14.

12 Berthoz A, Llinas R. Afferent neck projection to the cat cerebellar cortex. Exp Brain Res 1974;20:285-401.

${ }^{13}$ Gilman S, Bloedel JR, Lechtenberg R. Disorders of the Cerebellum. Philadelphia: Davis, 1981.

${ }^{14}$ Grant G. Spinocerebellar connections in the cat with particular emphasis on their cellular origin. Exp Brain Res Suppl 1982;6:466-75. 